www.refaad.com
المجلة الدولية للدراسات القانونية والفقهية المقارنة

International Journal of Legal and Comparative Jurisprudence Studies (LCJS)

Journal Homepage: https://www.refaad.com/views/LCJS/Home.aspx

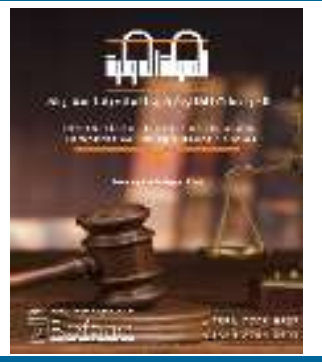

ISSN: 2708-6607(Online) 2708-6593(Print)

\title{
ضمانات تطبيق مبدأ الإجراءات الاحترازية في النزاعات المسلحة
}

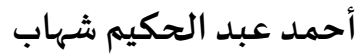

محامي- دكتوراه في القانون العام والقضاء- أستاذ القانون العام المساعد بكلية الشريعة والقانون - الجامعة الإسلامية بغزة- فلسطين shehab_ahmd@hotmail.com

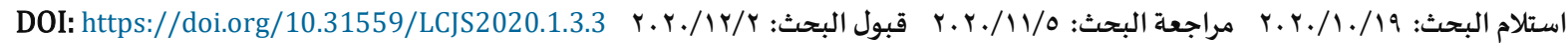

تعاني أحكام القانون الدولي عامة من إشكالية الفاعلية ومدى قدرة قواعد هذا القانون من فرض احترامه على المخاطبين بأحكامه،

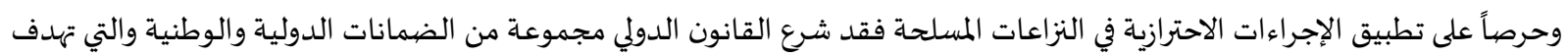

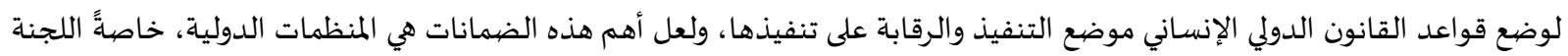

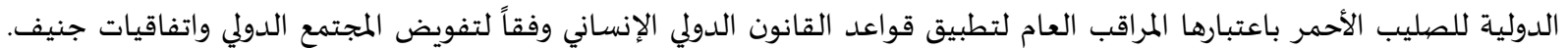

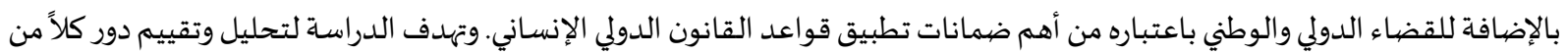

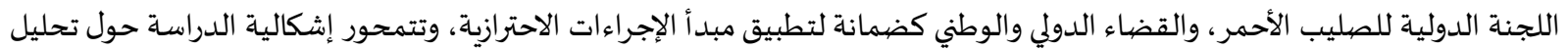

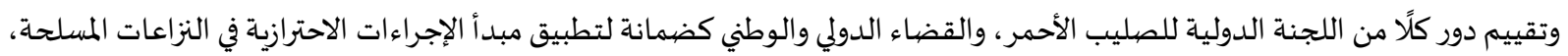
وإثراءً للدراسة فقد تناول الباحث بعض تورئ تطبيقات الإجراءات الاحترازية وقواعد الحظر في الشريعة الإسلامية. وتتبع الدراسة منهجية تحليلية في استعراض في التشريعات والاتفاقيات الدولية واستقصاء الشواهد التاريخية. وخلصت الدراسة لمجموعة من النتائج أهمها: تضطلع اللجنة الدولية للصليب الأحمر بدور هام في الرقابة على تطبيق مبدأ الإجراءات الاحترازية والرقابة عليه، بالإضافة للقضياء الدولي ممثلاً بالمحكمة الجنائية الدولية، والقضاء الوطني حال الانضمام لاتفاقيات جنيف ومواءمة التشريعات الوطنية معها. كما خلصت الدراسة لمجموعاة من

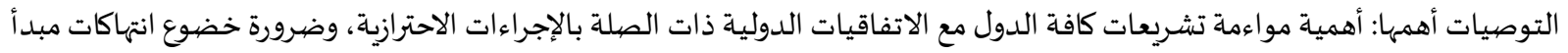

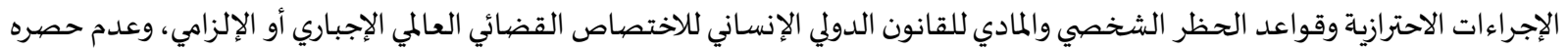
في محكمة معينة. الكلمات المفتاحية: الإجراءات الاحترازبة؛ الصليب الأحمر؛ القضاء الدولي؛ حقوق الإنسان.

ينطبق القانون الدولي الإنساني على أي وضع يدخل في أعمال القتال أو يترتب عليها، وقد اهتمت اتفاقيات جنيف لعام 9 أع 9 م والملحقان الإضيافيان لعام 9 VV إم بإقرار جملة من القواعد التي تهدف لإضفاء طابع إنساني على أي نزاع مسلح، بحيث لم يعد يقتصر انطباق أحكام القانون الدولي الإنساني

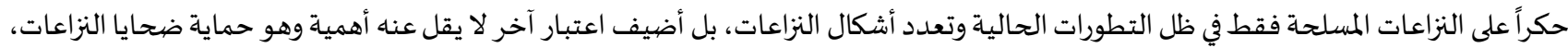
وبذلك يعد الإنسان القيمة العليا التي يتوجب الحفاظ عليها وحمايتها وهو ما أدى لتوسع النطاق الموضوعي لتطبيق القانون الدولي الإنساني. وتطبيقاً

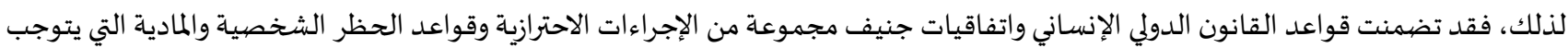
على أطراف النزاع اتخاذها قبل وأثناء وقوع النزاع لتجنب الانتهاكات الواقعة على الفئات والأعيان المحمية وفقاً للقانون الدولي والحد من استمرارها 
وقد ألزم القانون الدولي الإنساني والقانون الدولي لحقوق الإنسان الدول باحترام أحكام المعاهدات الدولية واتخاذ الإجراءات الكفيلة بتنفيذها،

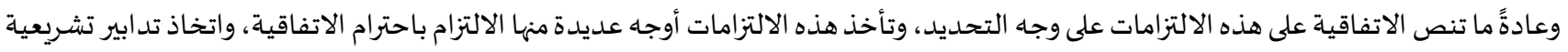

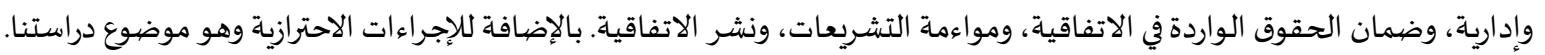
حيث تبرز أهمية الدراسة حول اظهار مدى قدرة وفاعلية قواعد القانون الدولي من فرض احترامه على المخاطبين بأحكامه، وإبراز ضمانانات تطبيق

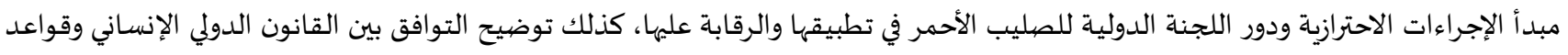

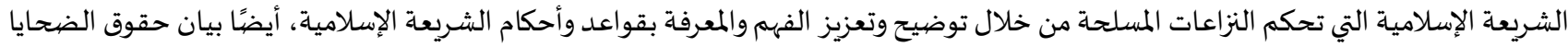
ووسائل حمايتهم في النزاعات المسلحة في القانون الدولي الإنساني والشريعة الإسلامياتة وتهدف الدراسة لتحليل وتقييم دور كلاً من اللجنة الدولية للصليب الأحمر، والقضياء الدولي والوطني كضمانة لتطبيق مبدأ الإجراءات الاحترازية،

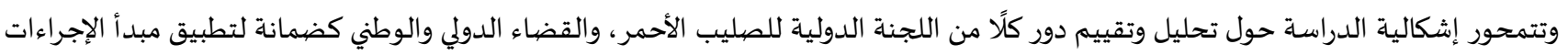
الاحترازية في النزاعات المسلحة، وإثراءً للدراسة فقد تناول الباحث بعض تطبيقات الإجراءات الاحترازية وقواعد الحظر في الشريعة الإسلامية. وقد تعرض الباحث في دراستاه الى مقدمة عامة حول موضوع الدراسة،، ثم عمل على تقسيم دراسته إلى محورين: تناول المحور الأول اهم الضمانيات

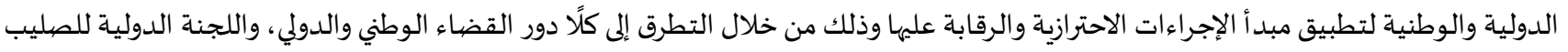

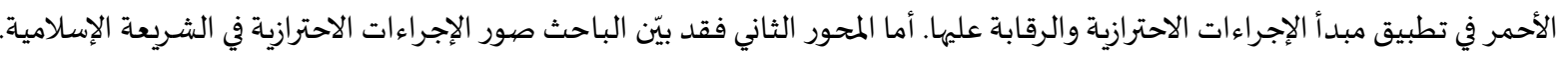

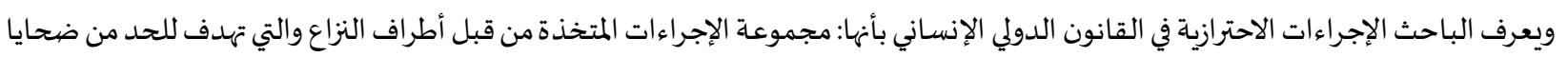

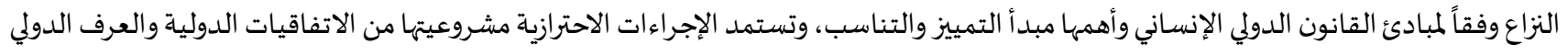
والدساتير الوطنياة. وبالحديث عن الإجراءات الاحترازية في القانون الدولي الإنساني، فلابد من الإشارة أن أهم مصادر القانون الدولي الإنساني هي المعاهدات الدولية

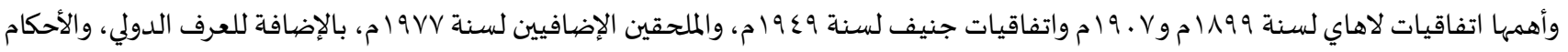
القضائية خاصيةً مع نشأة المحكمة الجنائية الدولية.

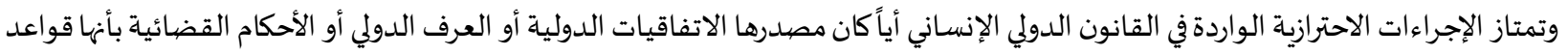

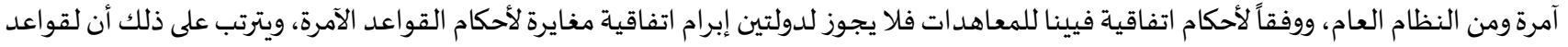

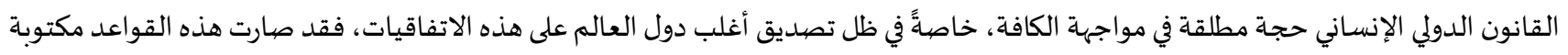

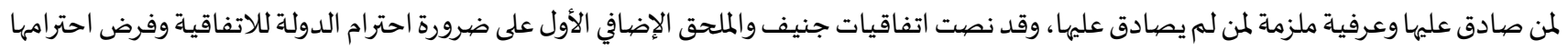

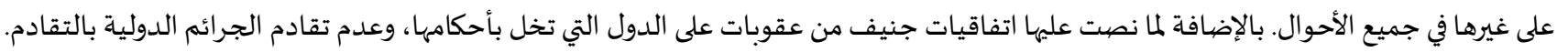

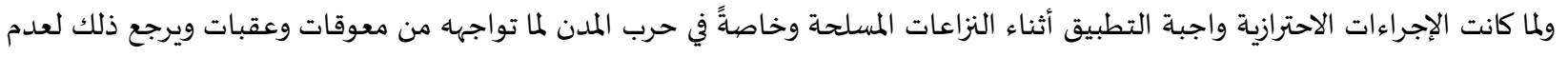

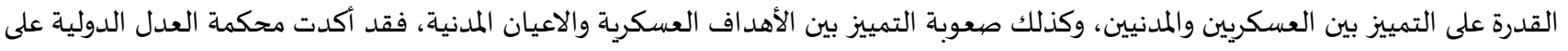

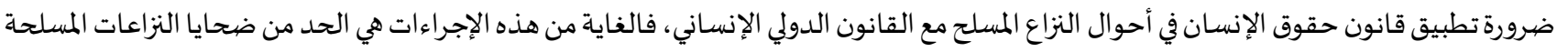

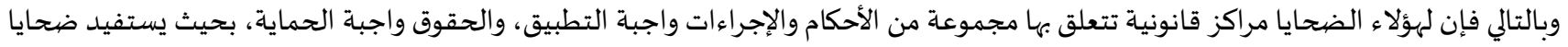

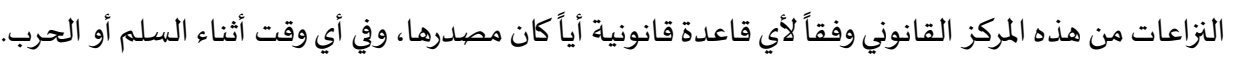

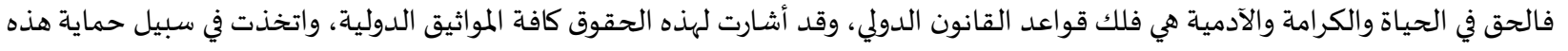

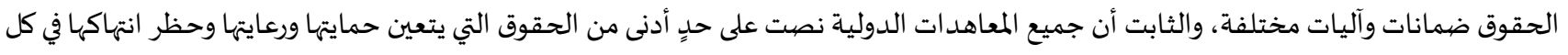
وقت وزمان ومكان. وقد نصت المادة المشتركة الثالثة من اتفاقيات جنيف على مجموعة من الأحكام والإجراءات التي تلتزم الأطراف المتعاقدة بتطبيقها في حال قيام نزاع

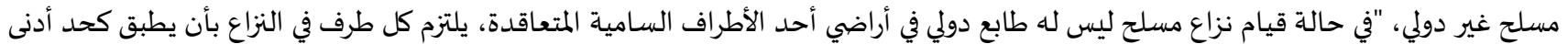

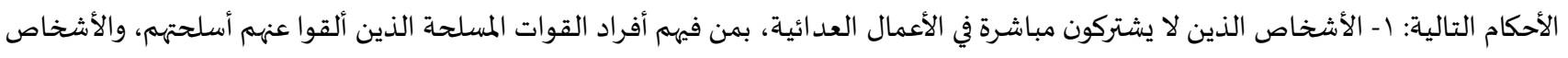

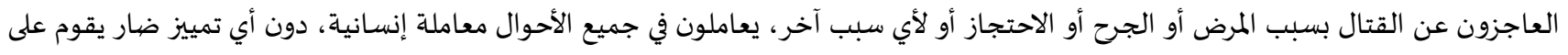

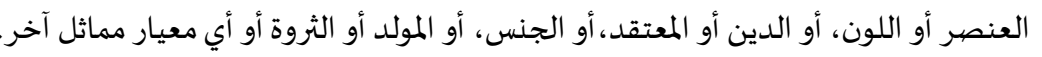

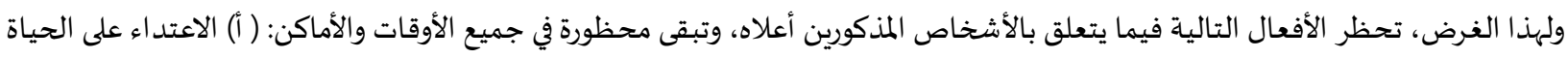

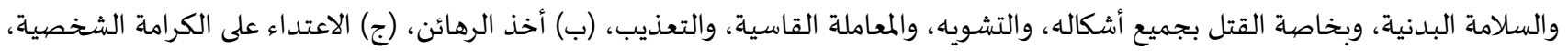

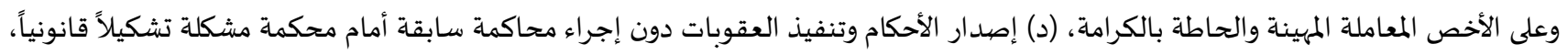
وتكفل جميع الضمانات القضائية اللازمة في نظر الشعوب المتمدنة. 
وعلى الأهمية التي جاءت بها المادة إلا أنها حصرت تطبيق الإجراءات الاحترازية بالأطراف الموقعة على اتفاقية جنيف، في حين أن ما يتفق مع روح

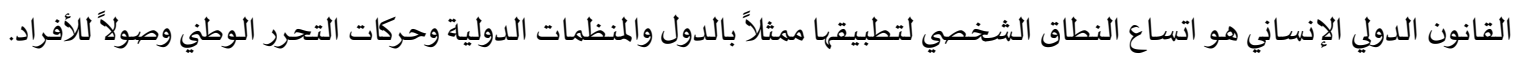

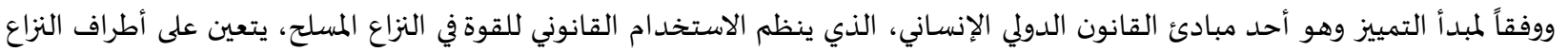

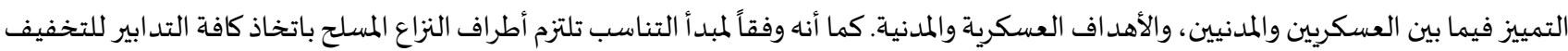

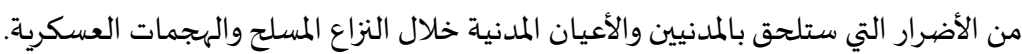
حيث صببتت اتفاقيات جنيف واللحقان الإضافيان قواعد القانون الدولي بالطابع الإنساني فلم يعد لأطراف النزاع مطلق الحريّة في اختيار وسائل

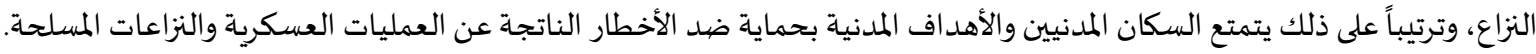
وللوقوف على الإجراءات الاحترازية الواجب اتخاذها وفقاً لمبدأ التمييز والتناسب قبل وأثناء وبعد النزاع المسلح فلابد من الرجباء الشخصي والموضيوعي للقانون الدولي الإنساني، والتي نورد منها على سبيل المثال لا الحصر. حظر منع الأجانب من مغادرة الإقليم أثناء النزاع' .

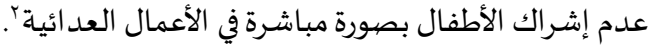
هالمعاملة الإنسانية ودون تمييز لكافة الأشخاص الذي يقعون في قبضة أحد أطراف النزاعَّ. توفير المؤن الغذائية والإمدادات الطبية وقبول أعمال الإغاثة من الصليب الأحمر أو أي منظمة إنسانية. يجب على السكان المدنيين رعاية الجرحى والمرضى والمنكوبين والبحث عن الموتى والإبلاغ عن أماكنهمء.

لا يجوز توقيع العقاب على أفراد البعثات الطبية أو إرغامهم على القيام بتصرفات تتنافي مع شرف المهنة؛. تلتزم الدول بكفالة حرية مرور جميع الأدوية والمهمات الطبية ومستلزمات العبادة والأغذية الضرورية والملابس للأطفال والنساء. حظر الهجوم العشوائي. حظر استهداف أفراد ومنشأت الخدمات الدينية والطبية والمستشفيات`. ضرورة تمييز معسكرات الاعتقال وتقديم الموقع الجغرافي للمعتقلات للدولة المعادية عن طريق الدولة الحاميةج. حظر استخدام المدنيين كدروع بشرية.

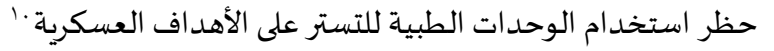

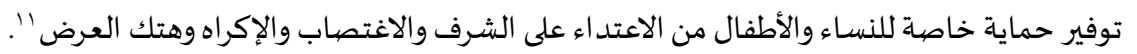
حظر التعدي على الممتلكات المدنية الخاصية والثقافية العامة.

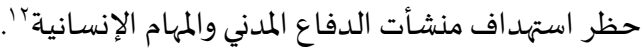
حظر مهاجمة الأعيان والمواد التي لا غنى لبقاء السكان عنها مثل السدود وشبكات الكهرباء والماء.

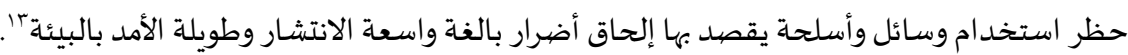

|تفاقية جنيف الرابعة (9 (19))، مادة (10).

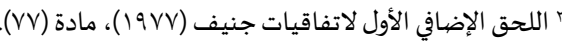

r اتفاقية جنيف الأولى (1999) )، مادة (r). ؛ اللحق الإضافي الأول لاتفاقيات جنيف (19VV) )، مادة (IVV) (IV).

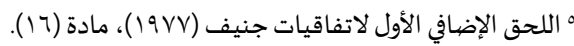

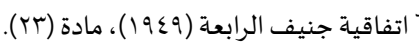

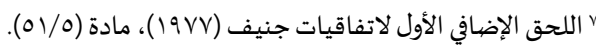

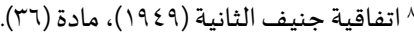

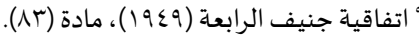

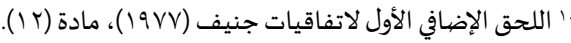

' اتفاقية جنيف الرابعة (19 (19)، مادة (YV).

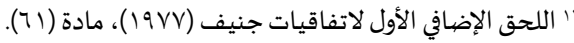

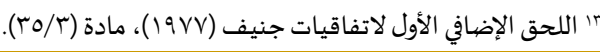


ضمانات تطبيق مبدأ الإجراءات الاحترازية:

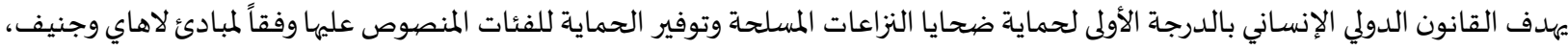

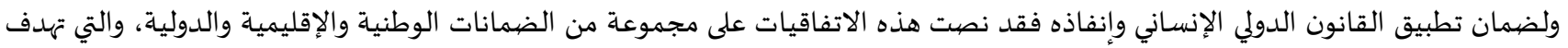

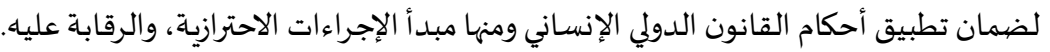

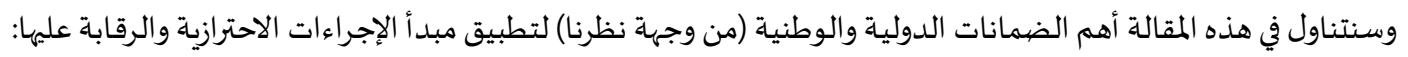

أولاً: دور القضياء الوطني والدولي في تطبيق مبدأ الإجراءات الاحترازية والرقابة عليها

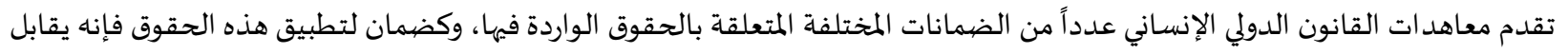

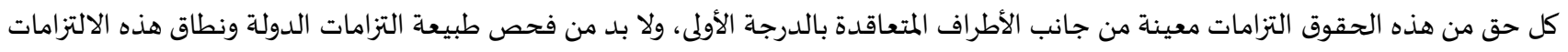

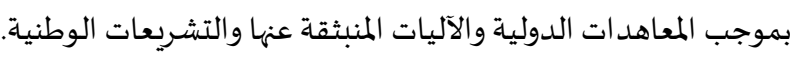

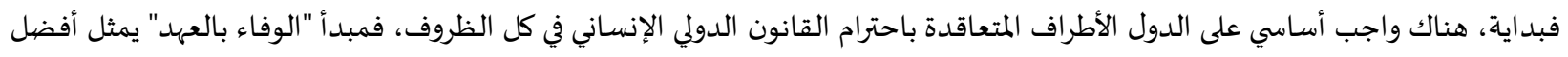

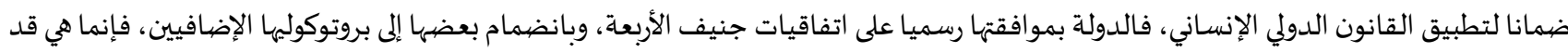

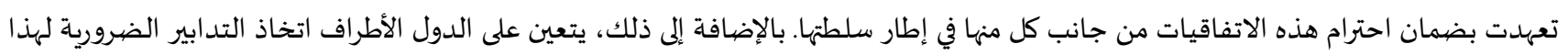

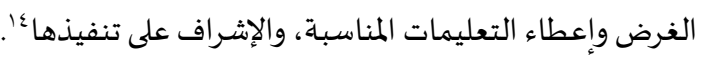

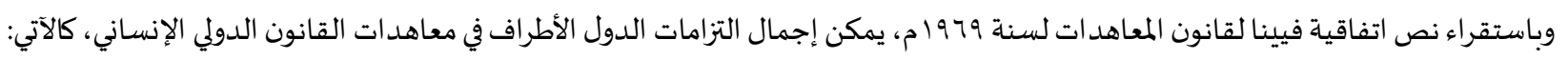

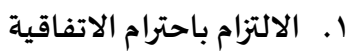

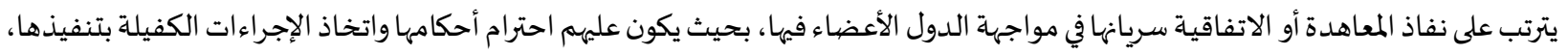

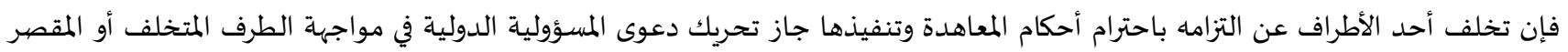
بالتزاماته 10

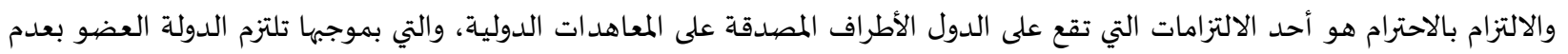

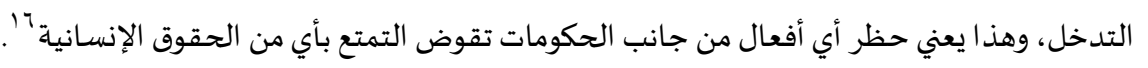

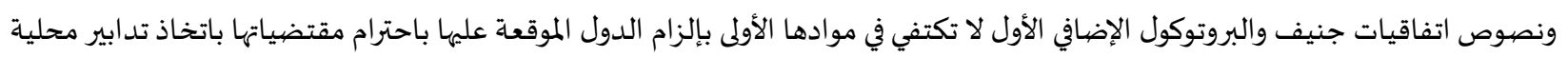

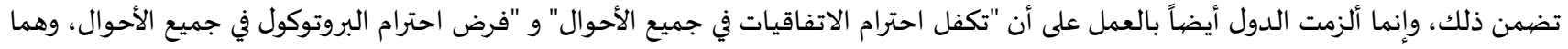

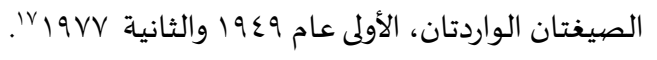

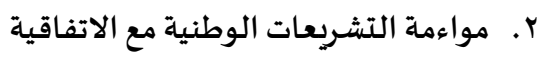

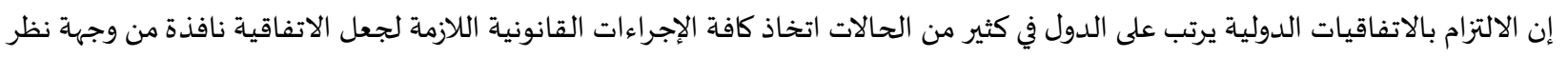

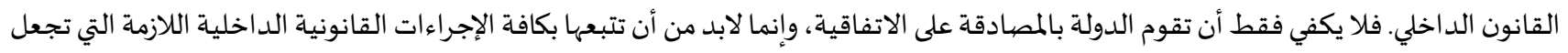

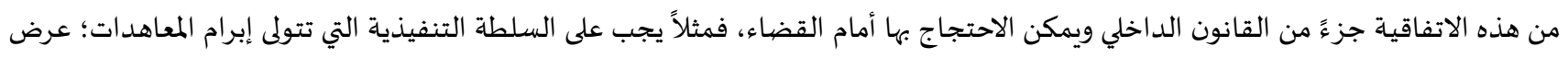

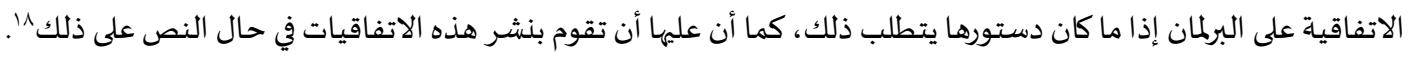

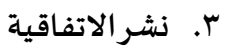

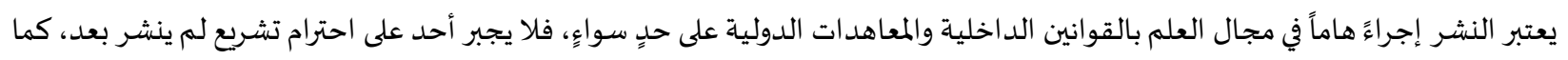

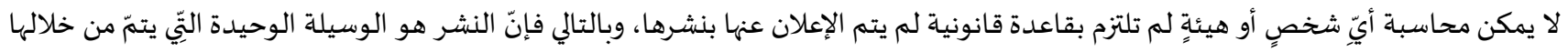

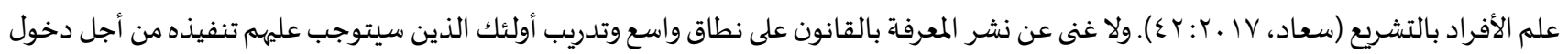

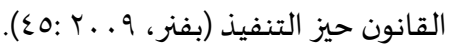

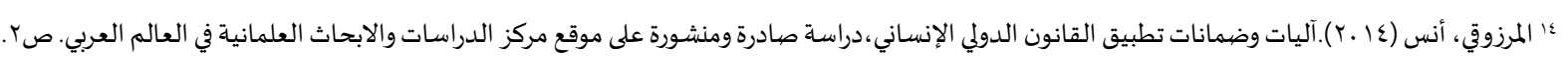
10

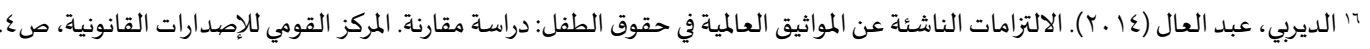

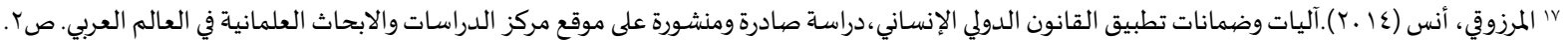

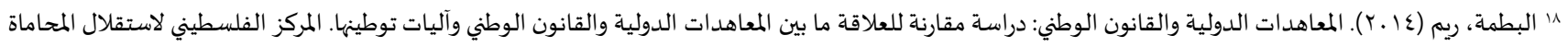

والقضاء، صبr. 


\section{ع. وضبع الحقوق الواردة في الاتفاقية موضع التنفيذ}

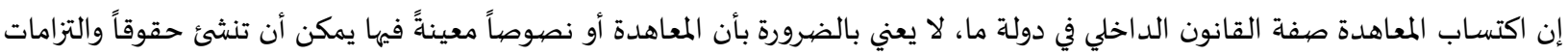

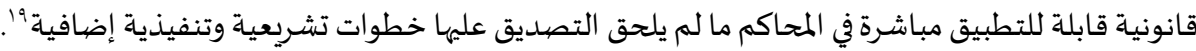

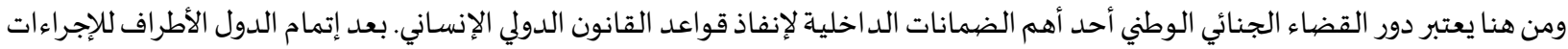

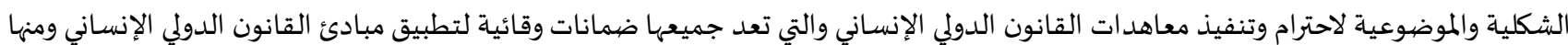
مبدأ الإجراءات الاحترازية.

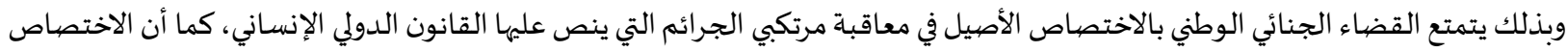

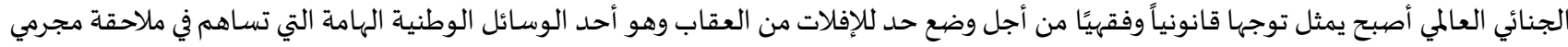

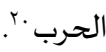

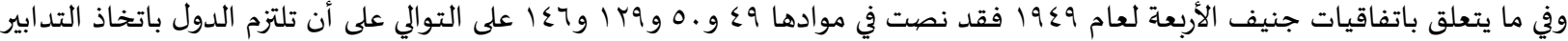

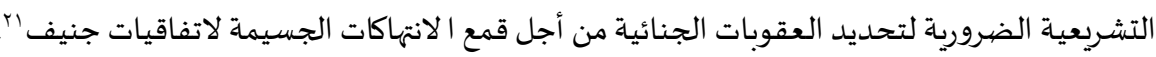

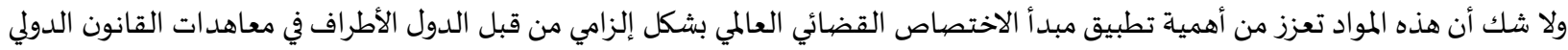

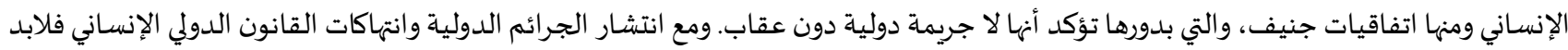

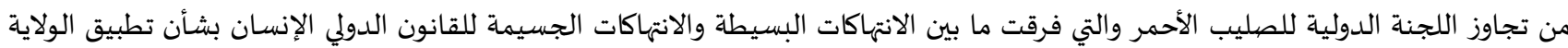
القضائية العالمياة.

بات يقينًا أنه لا مناص للحد من انتهاكات القانون الدولي الإنساني، والتي تزايدت وتيتها في الآونة الأخيرة، بسبب زيادة القوة التدميرية للأسلحة

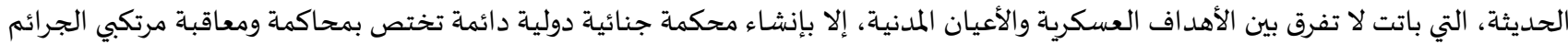

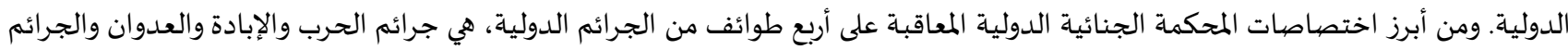

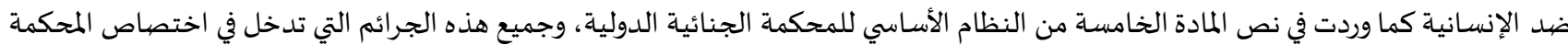

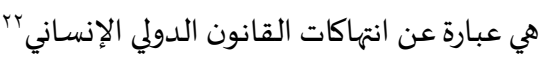

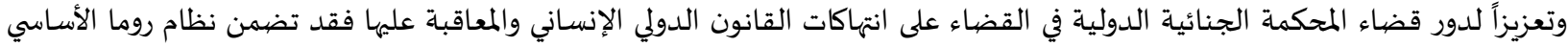

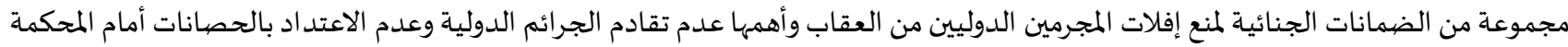
وإقرار مبدأ المسؤولية الشخصية.

ثانياً: دور اللجنة الدولية للصليب الأحمرفي تطبيق مبدأ الإجراءات الاحترازية والرقابة عليها

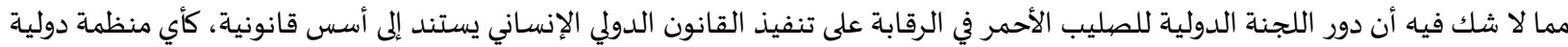

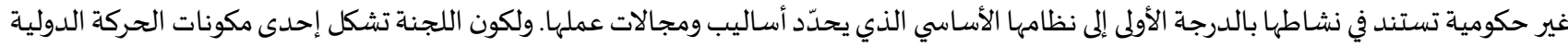

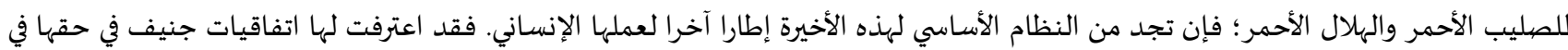

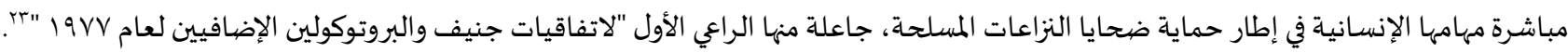

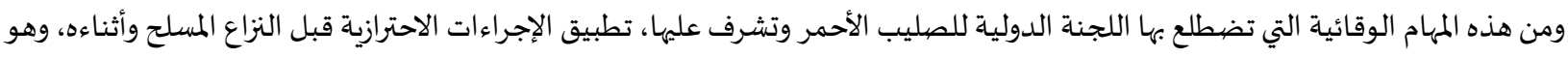
ما يقتضي التعاون مع أطراف النزاع للحد من ضحايا التمائ النزاعات المسلحة عبر تطبيق أهم مبادئ القانون الدولي الإنساني وهما مبدأ التمييز والتناسب.

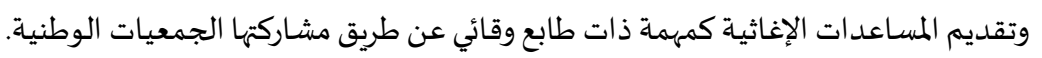

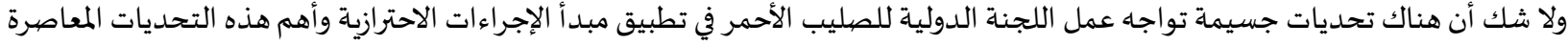

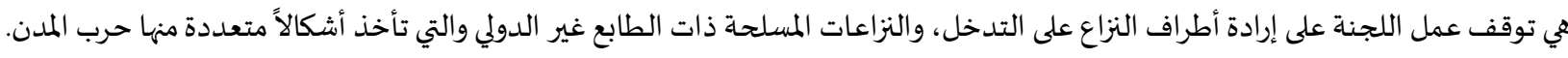

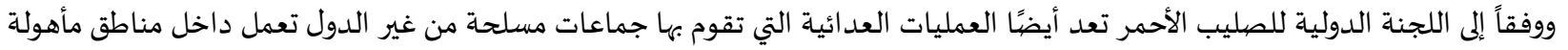

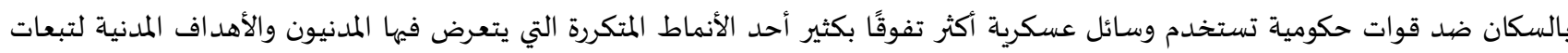

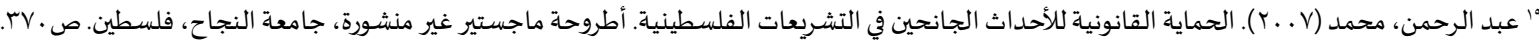

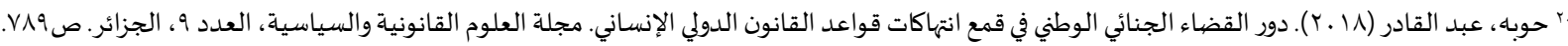

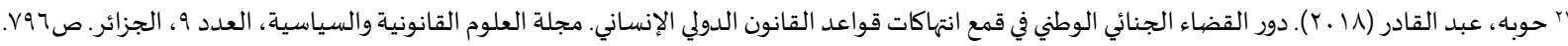

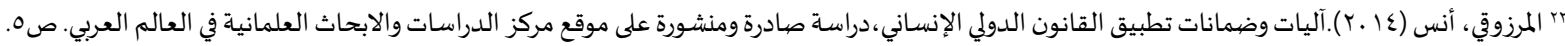

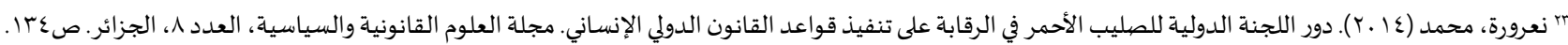


العمليات العدائية من أهم التحديات المعاصرة للقانون الدولي الإنسـاني گ وقد استغلت بعض الجيوش التداخل بين الجماعات المسلحة والمدنيين، الذي

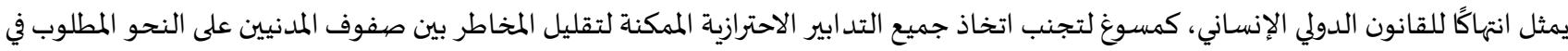

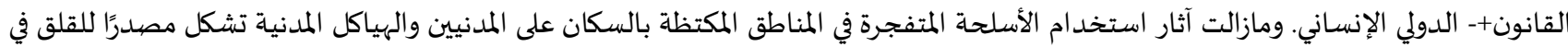

\section{صهور الإجراءات الاحترازية في الشريعة الإسلامية:}

إن مواثيق القانون الدولي الإنساني وضعت إداءل لكل فئة من الفئات المشمولة بالحماية نظاماً قانونياً محدداً، لكن منطلقات كل تلك المواثيق وأهد افها

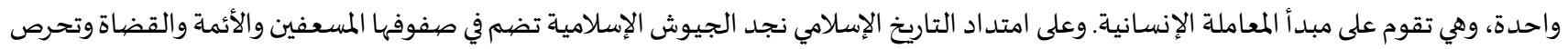

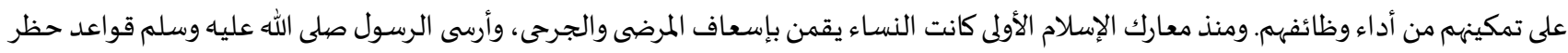

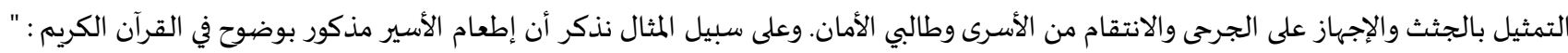

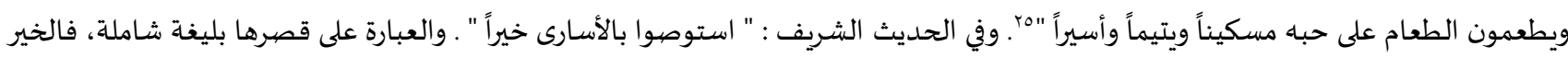

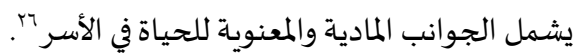

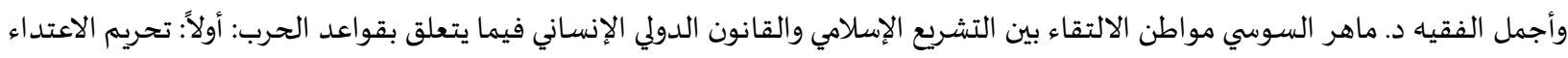

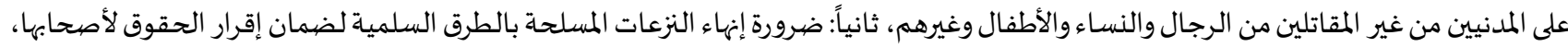

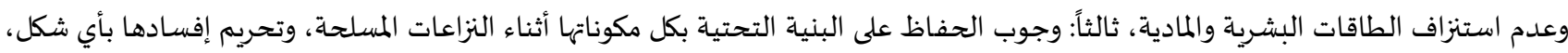

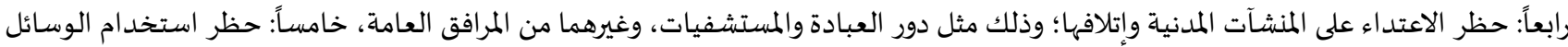

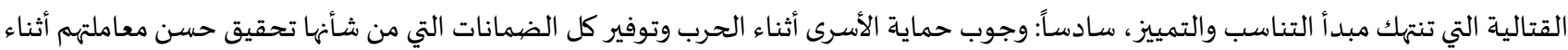
الأسر، سـابعاً: تحريم قتل الجرحى الذين عجزوا عن القتال، ووجوب مداواتهم والإحسان إلههم، ثامناً: تقرير المسؤولية على من ينتهك القواعد السابقة

ويعمل بخلاف مقتضياها'

والمتفحص لنصوص الشريعة الإسلامية يجد تطابق شبه تام من حيث المضهمون مع قواعد القانون الدولي الإنساني الخاصية بالنزاعات المسلحة

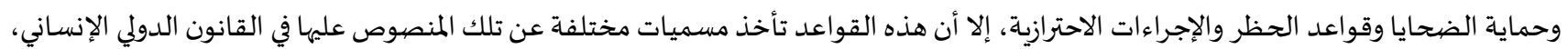

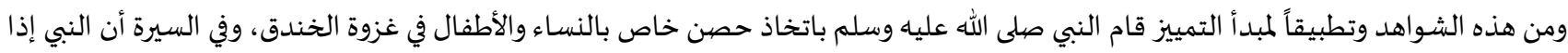

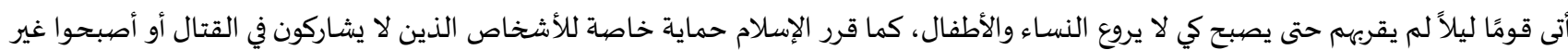

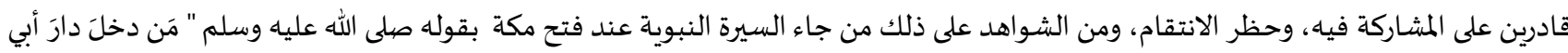

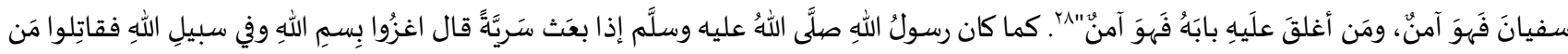

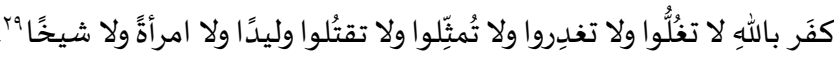

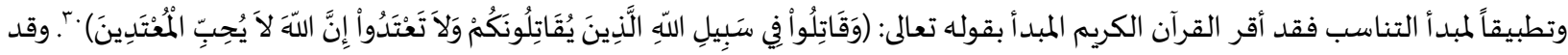

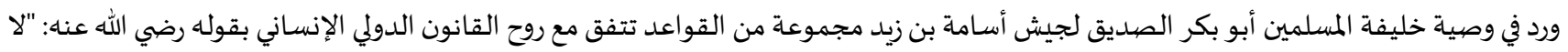

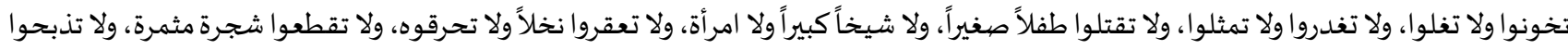

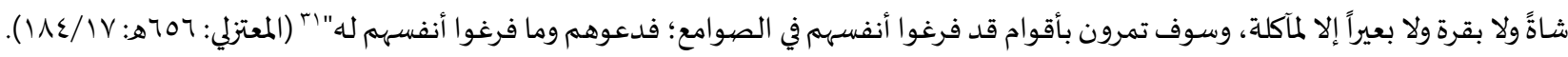

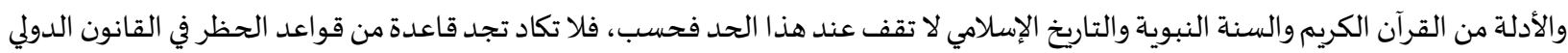

الإنساني إلا ولها أصل في الشريعة الإسلامية، وختاماً نورد هذه المقارنة بين قواعد القانون الدولي الإنساني والشريعة الإسلامية.

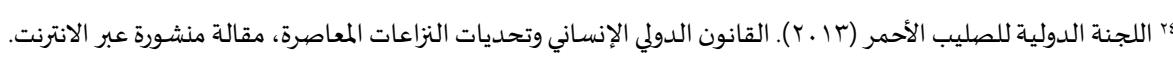

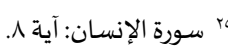

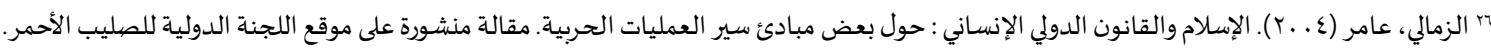

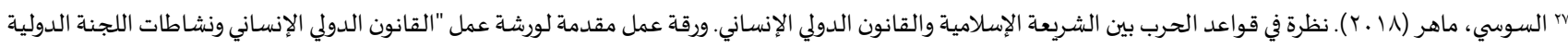

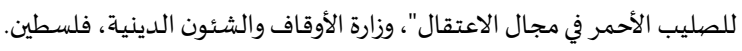

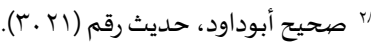

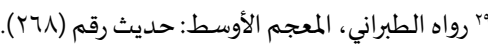

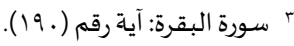

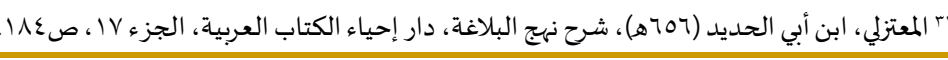


على سبيل المثال: تتضمن اتفاقيات جنيف لعام 9 أوبروتوكولاها الإضافيان لعام 19VV عدداً من الأحكام التي تحظر على نحو قاطع المعاملة

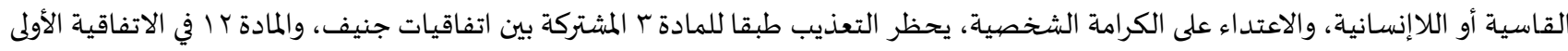

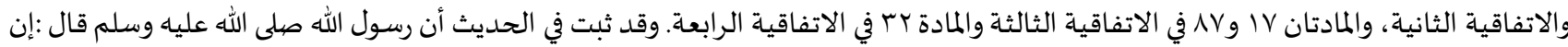
الله يعذب الذين يعذبون الناس في الدنيا .رواه مسلم. كما أجمع الفقه الإسلامي على أن التعذيب حرام، خاصة للمسجونين المتهمين.

الخاتمةة:

ناقشت هذه المقالة الإجراءات الاحترازية في ظل التحديات المرتبطة بالنزاعات المسلحة داخل المدن وأهم ضماناتها، متمثلة في دور القضياء الوطني

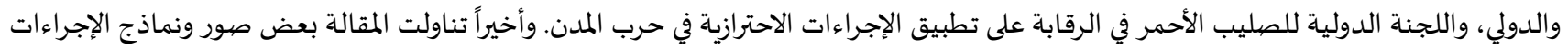
الاحترازية في الشريعة الإسلامية، وقد خلصت الدراسة لمجموعة من النتائج والتوصيات، وهي كالآتي:

النتائجج:

ا. ـ أن مبدأ الإجراءات الاحترازية مطبق في الشربعة الإسلامية بمسميات مختلفة عن القانون الدولي الإنسـاني.

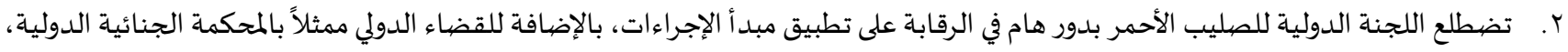

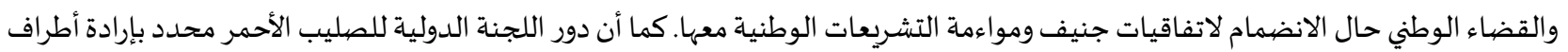
النزاع، وأنها لا تملك صاءحية مطلقة في تطبيق والرقابة على مبدأ الإجراءات الاحترازية.

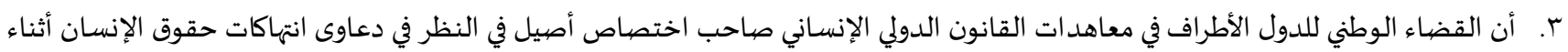
النزاعات المسلحة، وأن اختصاص القضاء الجنائي الدولي هو اختصاص تكميلي.

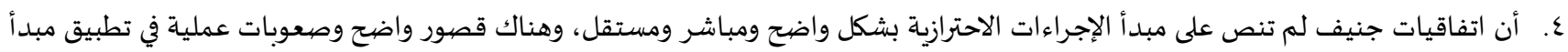

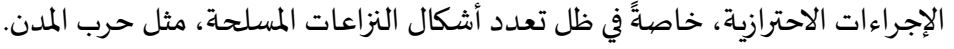

التوصيات: ا. أهمية مواءمة تشريعات كافة الدول مع الاتفاقيات الدولية ذات الصلة بالإجراءات الاحترازية، ومراجعة الاتفاقيات الدولية، خاصيةً اتفاقيات جنيف،

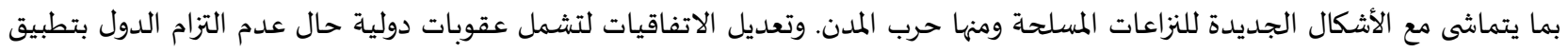

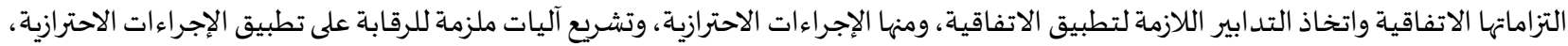
بحيث لا يتوقف عملها على إرادة أطراف النزاع.

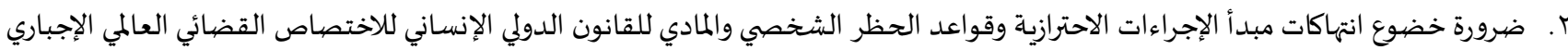
أو الإلزامي، وعدم حصره في محكمة معينة. ז. ضرورة إسهام كافة المؤسسات المعنية على الصعيد الوطني والمحلي بنشر وتعليم مبدأ الإجراءات الاحترازية، خاصيةً لأطراف النزاع، مثل الدول والجماعات المسلحة وحركات التحرر الوطني، وذلك من خلال تنسيق الجهود بين أشخاص القانون الدولي وتعزيز التعاون القضائي في سبيل الوصول

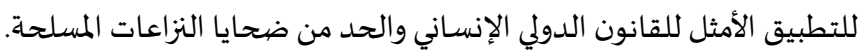

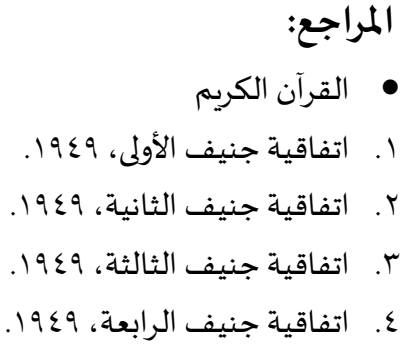

0. البطمة، ريم. (ع ا ـ r). "المعاهدات الدولية والقانون الوطني: دراسة مقارنة للعلاقة ما بين المعاهدات الدولية والقانون الوطني وآليات توطينها". المركز الفلسطيني لاستقلال المحاماة والقضاء، فلسطين.

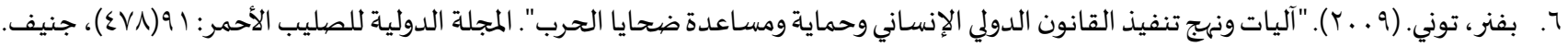

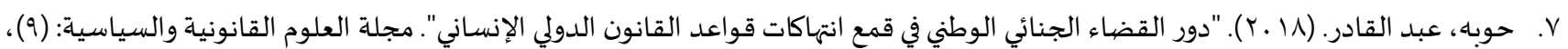
الجزائر.

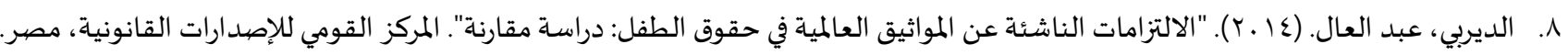


9. الزمالي، عامر. (ع . . (ا). "الإسلام والقانون الدولي الإنساني : حول بعض مبادئ سير العمليات الحربية". مقالة منشورة على موقع اللجنة الدولية

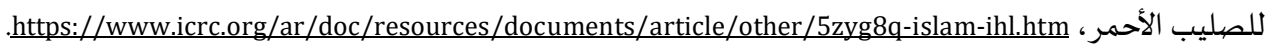

• ا. سعاد، رابح. (Y IV). "الجزائر والقانون الدولي لحقوق الإنسان". أطروحة دكتوراه غير منشورة. جامعة تلمسان، الجزائر. ا ا ـ السوسي، ماهر. (1) ـ Y). "نظرة في قواعد الحرب بين الشريعة الإسلامية والقانون الدولي الإنساني". ورقة عمل مقدمة لورشة عمل "القانون الدولي

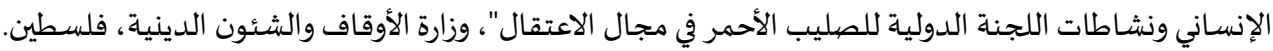

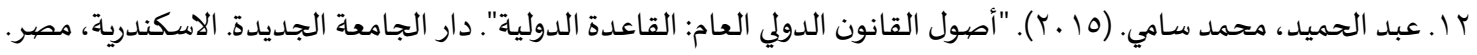

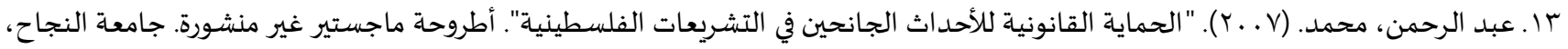
فلسطين. ع ا. اللجنة الدولية للصليب الأحمر. (با ـ ب). "القانون الدولي الإنساني وتحديات النزاعات المعاصرة". مقالة منشورة عبر الانترنت: https://www.icrc.org/ar/document/contemporary-challenges-ihl

0 ا ـ المرزوقي، أنس. (عـ ا ـ ا). "آليات وضمانات تطبيق القانون الدولي الإنساني". دراسة صادرة ومنشورة على موقع مركز الدراسات والابحاث العلمانية في

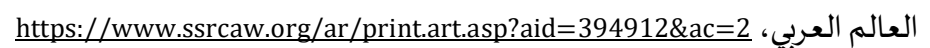

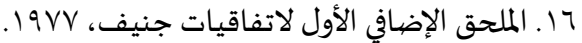

V ا. نعرورة، محمد. (ع ا ـا). "دور اللجنة الدولية للصليب الأحمر في الرقابة على تنفيذ قواعد القانون الدولي الإنساني". مجلة العلوم القانونية والسياسية: (م). الجزائر. 
www.refaad.com
المجلة الدولية للدراسـات القانونية والفقهية المقارنة

International Journal of Legal and Comparative Jurisprudence Studies (LCJS)

Journal Homepage: https://www.refaad.com/views/LCJS/Home.aspx

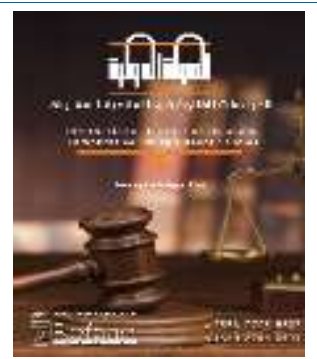

\title{
Guarantees of applying the principle of precautionary measures in armed conflicts
}

\author{
Ahmed Abdel Hakeem Shehab \\ Lawyer - PhD in Public Law and Judiciary, Assistant Professor of Public Law at the College of Sharia and Law, \\ Islamic University of Gaza, Palestine \\ shehab_ahmd@hotmail.com
}

Received: 19/10/2020 Revised: 5/11/2020 Accepted: 2/12/2020 DOI: https://doi.org/10.31559/LCJS2020.1.3.3

Abstract: The provisions of international law generally suffer from the problem of effectiveness and the ability of the provisions of this law to impose its respect on those addressing its provisions. In the interest of applying precautionary measures in armed conflicts, international law has enacted a set of international and national guarantees aimed at putting the provisions of international humanitarian law into effect and monitoring their implementation. Perhaps the most important of these guarantees are international organizations, especially the International Committee of the Red Cross as the general observer for the application of the provisions of international Humanitarian law in accordance with the mandate of the international community and the Geneva Conventions, in addition to the international and national judiciary as one of the most important guarantees for the application of the provisions of international humanitarian law. This article aims to analyze and evaluate the role of the International Committee of the Red Cross and the international and national judiciary as a guarantee for the application of the precautionary measure's principle. To enrich the study, the researcher addressed some applications of precautionary measures and prohibition rules in Islamic sharia. The study follows an analytical methodology in reviewing international legislation and conventions as well as in investigating historical evidence.

The study reached a set of results, the most important of which are: The International Committee of the Red Cross plays an important role in monitoring and supervising the application of the principle of precautionary measures, in addition to the international judiciary represented by the International Criminal Court as well as the national judiciary when it accedes to the Geneva Conventions and harmonizes national legislations with them. The study is concluded with a set of recommendations, the most important of which are: the importance of harmonizing the legislations of all countries with international conventions that are related to the precautionary measures, and the necessity for the subjection of the violations of the principle of precautionary measures and the rules of the international humanitarian law to a compulsory universal jurisdiction, not to limit it to a specific court.

Keywords: precautionary measures; the Red Cross; international judiciary; human rights.

\section{References:}

[1] 'bd Alhmyd, Mhmd Samy. (2015). "Aswl Alqanwn Aldwly Al'am: Alqa'dh Aldwlyh". Dar Aljam'h Aljdydh. Alaskndryh, Msr.

[2] 'bd Alrhmn, Mhmd. (2007). "Alhmayh Alqanwnyh Llahdath Aljanhyn Fy Altshry'at Alflstynyh". Atrwht Majstyr Ghyr Mnshwrh. Jam't Alnjah, Flstyn.

[3] Atfaqyh Jnyf Alawla, 1949.

[4] Atfaqyh Jnyf Althanyh, 1949.

[5] Atfaqyh Jnyf Althalthh, 1949. 
[6] Atfaqyh Jnyf Alrab'eh, 1949.

[7] Albtmh, Rym. (2014). "Alm'ahdat Aldwlyh Walqanwn Alwtny: Drash Mqarnh Ll'laqh Ma Byn Alm'ahdat Aldwlyh Walqanwn Alwtny Walyat Twtynha". Almrkz Alflstyny Lastqlal Almhamah Walqda', Flstyn.

[8] Bfnr, Twny. (2009). "Alyat Wnhj Tnfyd Alqanwn Aldwly Alensany Whmayh Wmsaedt Dhaya Alhrb". Almjlh Aldwlyh Llslyb Alahmr: 91(478), Jnyf.

[9] Aldyrby, 'bd Al'al. (2014). "Alaltzamat Alnash'h 'n Almwathyq Al'almyh Fy Hqwq Altfl: Drash Mqarnh". Almrkz Alqwmy Llesdarat Alqanwnyh, Msr.

[10] Hwbh, 'bd Alqadr. (2018). "Dwr Alqda' Aljna'y Alwtny Fy Qm' Anthakat Qwa'd Alqanwn Aldwly Alensany". Mjlt Al'lwm Alqanwnyh Walsyasyh: (9), Aljza'r.

[11] Alljnh Aldwlyh Llslyb Alahmr. (2013). "Alqanwn Aldwly Alensany Wthdyat Alnza'at Alm'asrh". Mqalh Mnshwrh 'br Alantrnt: https://www.icrc.org/ar/document/contemporary-challenges-ihl.

[12] Almrzwqy, Ans. (2014). "Alyat Wdmanat Ttbyq Alqanwn Aldwly Alensany". Drash Sadrh Wmnshwrh 'la Mwq' Mrkz Aldrasat Walabhath Al'Imanyh Fy Al'alm Al'rby, https://www.ssrcaw.org/ar/print.art.asp?aid=394912\&ac=2

[13] Almlhq Aledafy Alawl Latfaqyat Jnyf, 1977.

[14] N'rwrh, Mhmd. (2014). "Dwr Alljnh Aldwlyh Llslyb Alahmr Fy Alrqabh 'la Tnfyd Qwa'd Alqanwn Aldwly Alensany". Mjlt Al'lwm Alqanwnyh Walsyasyh: (8). Aljza'r.

[15] S'ead, Rabh. (2017). "Aljza'r Walqanwn Aldwly Lhqwq Alensan". Atrwht Dktwrah Ghyr Mnshwrh. Jam't Tlmsan, Aljza'r.

[16] Alswsy, Mahr. (2018). "Nzrh Fy Qwa'd Alhrb Byn Alshry'h Aleslamyh Walqanwn Aldwly Alensany". Wrqh 'ml Mqdmh Lwrshh 'ml "Alqanwn Aldwly Alensany Wnshatat Alljnh Aldwlyh Llslyb Alahmr Fy Mjal Ala'tqal", Wzart Alawqaf Walsh'wn Aldynyh, Flstyn.

[17] Alzmaly, 'amr. (2004). "Aleslam Walqanwn Aldwly Alensany: Hwl B'd Mbad' Syr Al'mlyat Alhrbyh". Mqalh Mnshwrh 'la Mwq' Alljnh Aldwlyh Llslyb Alahmr, https://www.icrc.org/ar/doc/resources/documents/article/other/5zyg8q-islam-ihl.htm. 